\title{
Phenotyping and QTL mapping for cold tolerance at the germination and seedling stages in Argentine temperate rice
}

\author{
Martha Lucrecia Bonell (D) - Gustavo Gabriel Arguissain • María Andrea Crepy • \\ Paula del Carmen Fernández • Ruth Amelia Heinz
}

Received: 4 October 2019/Accepted: 9 July 2020/Published online: 19 July 2020

(C) Springer Nature B.V. 2020

\begin{abstract}
In Argentina, the yield potential of rice (Oryza sativa L.) under suboptimal temperatures of temperate regions and during early sowing in tropical regions depends on its low-temperature germinability (LTG) and subsequent cold tolerance at the seedling stage (CTS). This study focused on the phenotyping and identification of contrasting henotypes for LTG and CTS of 18 rice genotypes genotyped with 49 markers previously linked to cold tolerance. The selected markers revealed phenotypic variability among the genotypes evaluated. The results also demonstrated that both traits are regulated by different genes and that CTS is associated with differential alleles of candidate gene (CG)-based markers. Biplot
\end{abstract}

Electronic supplementary material The online version of this article (https://doi.org/10.1007/s10681-020-02661-3) contains supplementary material, which is available to authorized users.

M. L. Bonell ( $₫)$ · G. G. Arguissain · M. A. Crepy Estación Experimental Agropecuaria Concepción del Uruguay, Instituto Nacional de Tecnología Agropecuaria, Ruta 39 Km 143,5, CC 6, 3260 Concepción del Uruguay, Entre Ríos, Argentina

e-mail: bonell.martha@inta.gob.ar

P. del Carmen Fernández · R. A. Heinz

Instituto de Agrobiotecnología y Biología Molecular IABIMO - (INTA-CONICET), Instituto de Biotecnología, Centro de Investigaciones en Ciencias Agronómicas Y Veterinarias (CICVyA), Instituto Nacional de Tecnología Agropecuaria, Calle Repetto y De Los Reseros S/N, C.P. 1686 Hurlingham, Buenos Aires, Argentina clustering analysis allowed the selection of two cultivars, H298a/90 and Puitá INTA CL, contrasting for LTG, CTS and CG alleles. An $\mathrm{F}_{2}$ population $(n=128)$ derived from these two cultivars was genotyped with 43 SSR- and CG-based markers and $89 \mathrm{~F}_{2: 3}$ families ( $\sim 1800$ seedlings) phenotyped for CTS. Single-marker analysis detected two major QTL that had been previously reported for CTS: qCTS11, which explained $18.35 \%$ of the phenotypic variance at 7 days of treatment, and $q$ CTS12, which explained $26.62 \%$ and $47.94 \%$ of the phenotypic variance at 7 and 14 days of treatment, respectively. Marker-assisted introgression of QTL alleles favorable for CTS into Puitá INTA CL, which has high LTG, could contribute to obtaining breeding lines with early seedling vigor adapted to the sowing season in Argentina.

Keywords Cold tolerance (CT) - Low-temperature germination ability (LTG) - Cold tolerance at seedling stage (CTS) · Candidate genes · Marker-assisted selection (MAS)

\section{Introduction}

Rice (Oryza sativa L.) grows in several ecological conditions and environments, even under abiotic stresses such as low temperatures. However, temperatures below $20{ }^{\circ} \mathrm{C}$ decrease the speed and percentage 
of germination, survival, and seedling growth, all of which results in lower early seedling vigor (Mahender et al. 2015). The japonica and indica rice cultivars differ in their cold tolerance (CT) and are generally adapted to temperate and tropical regions, respectively (Mahender et al. 2015). In countries with temperatures that are suboptimal for rice growth such as Brazil, Uruguay and Argentina, in the past, rice production was based on US japonica cultivars, which have good grain quality but low yield potential, whereas the current rice production is based mainly on indica cultivars, which have higher yield potential with improved grain quality (Cruz et al. 2013; Martínez et al. 2014). However, in the rice-growing areas of Argentina, the average minimum temperature during typical or early sowing ranges between 7 and $16{ }^{\circ} \mathrm{C}$ (INTA 2019), and thus indica cultivars suffer from cold stress. Thus, the establishment of a successful direct-seeded rice crop requires the development of improved cultivars with low-temperature germinability (LTG) and high CT at the seedling stage (CTS).

Traditional breeding approaches include the evaluation of genetic variability for CT under field or controlled conditions and the identification of superior breeding lines. (Cruz et al. 2013; Zhang et al. 2014). However, it is difficult to develop simple and standardized methods because researchers establish different evaluation times, temperatures, and indices for evaluation of CT according to the developmental stage (Zhang et al. 2014). For example, at the germination stage, researchers calculate the germination percentage, germination rate, or germination vigor and record the length or dry weight of the coleoptile (Cruz and Milach 2004; Ye et al. 2009), whereas, at the seedling stage, researchers determine the degree of damage by determining a visual score of yellowing and wilting and by determining the seedling survival rate and growth (Bertin et al. 1996; Yoshida et al. 1996; Kim and Tai 2011). Other important characteristics usually determined include physiological measurements such as electrolyte leakage, chlorophyll and proline content, and activity of the antioxidant system (Huang and Guo 2005; Kim et al. 2011; Zhang et al. 2014).

Genes involved in CT include genes linked to perception and signal transduction (e.g. transcription factors and kinases), synthesis of lipid desaturases and antifreeze and osmoprotectant solutes (e.g. proline), and detoxification (e.g., glutathione S-transferase, superoxide dismutase, ascorbate peroxidase) (Mizoi and Yamaguchi-Shinozaki 2013; Janmohammadi et al. 2015; Sperotto et al. 2017). This reflects the complex nature of the CT trait, which involves multiple metabolic pathways and genes, which are controlled mostly by temperature but also affected by other abiotic stresses, such as drought and salt.

Different breeding studies have reported the existence of major genes and quantitative trait loci (QTL) for LTG and CTS (Zhi-Hong et al. 2005; Andaya and Tai 2006; Fujino and Matsuda 2010; Shakiba et al. 2017). Nevertheless, since the reported DNA polymorphisms linked to these QTL traits are specific for each population used for mapping, the validation of markers in different populations and/or environments is always essential (Collard et al. 2008; Hospital 2009). For this validation, breeding programs routinely follow strategies to exploit the mapped QTL in diverse germplasms. Major QTL (i.e. QTL with large estimated effects) are particularly well adapted to fit into small breeding programs, because they can be easily introgressed by standard breeding procedures to obtain improved cultivars (Bernardo 2008).

Many of the most widespread indica cultivars currently used in countries of South America have been developed by national breeding programs such as EMBRAPA and IRGA in Brazil, INIA in Uruguay, and INTA in Argentina (Martinez et al. 2014). The INTA breeding program has a history of more than 40 years of crossbreeding and selection for yield potential, grain quality (mostly long grain), and resistance to diseases and herbicides. In the last three decades, several cultivars, including CT6919-INTA, Cambá INTA, Tranquilo-FL-INTA, and the imidazolinone-tolerant cultivars Puitá INTA CL and Gurí INTA CL, have been registered and are now widely sown not only in Argentina but also in Brazil and other countries. However, because the INTA breeding program has an indica genetic background, it is still necessary to improve the CT of their germplasm. Thus, the aim of the present study was to identify a consistent method to assess the phenotypic variability for LTG and CTS of Argentine rice germplasms and analyze their genetic diversity at simple sequence repeat SSR- and CG-based markers previously associated with CT. In addition, we clustered and selected contrasting genotypes at the phenotypic and genotypic level to develop a bi-parental segregating population, 
and mapped QTL for CTS for their introgression in genotypes with high LTG.

\section{Materials and methods}

Plant material

The phenotypic variability for LTG and CTS and the genetic diversity at SSR- and CG-based markers linked to CT were assessed with a mini-core set of 18 genotypes. Nine of these genotypes were control cultivars (both indica and japonica) for cold susceptibility and tolerance at the germination and/or seedling stages (Table 1). The remaining nine genotypes were the Argentine indica cultivars Puitá INTA CL and Cambá INTA CL and breeding lines originating from crosses involving indica and japonica genotypes.

\section{Phenotyping for LTG and CTS}

LTG screening was performed by first treating seeds, which had been harvested in the same cropping season, at $50{ }^{\circ} \mathrm{C}$ for 5 days to break dormancy (Sthapit and Whitcombe 1998). Seed quality was evaluated at $28{ }^{\circ} \mathrm{C}$ and the selected seed samples of each genotype showed $100 \%$ of germination by the fifth day of incubation. Then, seeds selected for size uniformity and absence of spots were sterilized with ethanol $70 \%$ for $30 \mathrm{~s}$, sodium hypochlorite $10 \%$ for $20 \mathrm{~min}$ and washed three times with sterile distilled water. Seeds $(\mathrm{n}=50)$ were placed on Petri dishes containing germination paper with $6 \mathrm{ml}$ of sterile water and incubated at $16{ }^{\circ} \mathrm{C}$ in darkness. The experiment was conducted in a completely randomized design with four replicates for each genotype. The following germination indices were calculated:

- Germination percentage $(\mathrm{GP})=(\mathrm{Ni} / 50) * 100$, where $\mathrm{Ni}$ is the number of seeds germinated at 4 , 7 and 14 days of treatment.

- Germination vigor $(\mathrm{GV})=\mathrm{N} 4 / 4+\mathrm{N} 7 / 7+\mathrm{N} 14 /$ 14 , where $\mathrm{N} 4$ is the number of seeds germinated at 4 days of treatment, and N7 and N14 are the numbers of newly germinated seeds at 7 and 14 days of treatment (Maguire 1962).

- Germination rate (GR) was calculated according to Krishnasamy and Seshu (1989) as GR1 $=($ N4/N7) $* 100$ and GR2 $=(\mathrm{N} 4 / \mathrm{N} 14) * 100$, where N4, N7 and N14 are the total numbers of seeds germinated at 4, 7 and 14 days. This index was also calculated according to Sthapit and Witcombe (1998) as
Table 1 Origin, subspecies and type of germplasm of 18 rice genotypes and their low-temperature germinability (LTG) and cold tolerance at seedling stage (CTS)

${ }^{\mathrm{a}}$ Subspecies of rice: mixed genotype derived from japonica $\mathrm{x}$ indica crosses

${ }^{\mathrm{b}, \mathrm{c}}$ LTG and CTS evaluation under several lowtemperature conditions (Cruz and Milach 2004; Sharifi 2010; Andaya and Mackill 2003a, b; Kim and Tai 2011) classified as $S$ susceptible, $T$ tolerant, $U$ Unknown

\begin{tabular}{llllll}
\hline Genotype & Origin & Subsp $^{\mathrm{a}}$ & Germplasm & LTG $^{\text {b }}$ & CTS $^{\mathrm{c}}$ \\
\hline IRGA 409 & Brazil & Indica & Cultivar & $\mathrm{S}$ & $\mathrm{S}$ \\
IRGA 417 & Brazil & Indica & Cultivar & $\mathrm{S}$ & $\mathrm{S}$ \\
El Paso 144 & Brazil & Indica & Cultivar & $\mathrm{S}$ & $\mathrm{S}$ \\
IR50 & Philippines & Indica & Cultivar & $\mathrm{S}$ & $\mathrm{S}$ \\
Silewah & Indonesia & Japonica & Cultivar & $\mathrm{U}$ & $\mathrm{T}$ \\
L-202 & USA & Japonica & Cultivar & $\mathrm{T}$ & $\mathrm{T}$ \\
M-202 & USA & Japonica & Cultivar & $\mathrm{U}$ & $\mathrm{T}$ \\
Somewake & Japan & Japonica & Cultivar & $\mathrm{U}$ & $\mathrm{T}$ \\
Koshihikari & Japan & Japonica & Cultivar & $\mathrm{T}$ & $\mathrm{U}$ \\
Puitá INTA CL & Argentina & Indica & Cultivar & $\mathrm{U}$ & $\mathrm{U}$ \\
Cambá INTA & Argentina & Mixed & Cultivar & $\mathrm{U}$ & $\mathrm{U}$ \\
Ant11786 & Argentina & Mixed & Breeding line & $\mathrm{U}$ & $\mathrm{U}$ \\
ECR61 & Argentina & Mixed & Breeding line & $\mathrm{U}$ & $\mathrm{U}$ \\
ECR57 & Argentina & Mixed & Breeding line & $\mathrm{U}$ & $\mathrm{U}$ \\
H298a/90 & Argentina & Mixed & Breeding line & $\mathrm{U}$ & $\mathrm{U}$ \\
CR675 & Argentina & Mixed & Breeding line & $\mathrm{U}$ & $\mathrm{U}$ \\
ECR153 & Argentina & Mixed & Breeding line & $\mathrm{U}$ & $\mathrm{U}$ \\
ECR67 & Argentina & Mixed & Breeding line & $\mathrm{U}$ & $\mathrm{U}$ \\
\hline
\end{tabular}


$\mathrm{GR} 3=(((\mathrm{N} 4+\mathrm{N} 7) / 2) / 50) * 100$ and $\mathrm{GR} 4=((($ $\mathrm{N} 4+\mathrm{N} 7+\mathrm{N} 14) / 3) / 50) * 100$.

At 14 days of treatment, the conditions were changed to a photoperiod of $12 \mathrm{~h}$ light/12 $\mathrm{h}$ darkness at $16{ }^{\circ} \mathrm{C}$ for a total of 28 days. A visual rating based on size and development was evaluated by recording the percentage of seedlings with shoots both smaller and larger than $0.5 \mathrm{~cm}(\% \mathrm{~S}<0.5$ and $\% \mathrm{~S}>0.5$ respectively), normally developed seedlings (\%NS), and remaining ungerminated seeds (\%UG). CT was evaluated with a visual scale of 1 (tolerant, green color) to 9 (susceptible, yellow color, stunted growth or wilting). Seedling growth was estimated by dry matter. For this purpose, the resulting weights of samples dried at $65{ }^{\circ} \mathrm{C}$ for 3 days were recorded. Dry weight (mg) of the shoots (DWS), dry weight of the roots (DWR) and total dry weight (DWT) were determined for each Petri dish. The number of normal seedlings (S) was counted and then the average dry weight of seedlings was calculated as DWS/S, DWR/S and DWT/S.

CTS was screened by sowing seeds in trays containing sterile soil and perlite $(70: 30 \mathrm{v} / \mathrm{v})$ and incubating at $28{ }^{\circ} \mathrm{C}$ with $12 \mathrm{~h} \mathrm{light} / 12 \mathrm{~h}$ darkness in a chamber (Conviron SH10, Canada) until the seedlings reached the 3-4 leaf stage. Subsequently, the temperature was lowered to $9{ }^{\circ} \mathrm{C}$ and two replicates containing 40 seedlings per genotype were evaluated for $\mathrm{CT}$ by using a visual scale $(1=$ dark green seedlings, $3=$ light green seedlings, $5=$ yellow seedlings, 7 = brown seedlings, $9=$ dead seedlings) according to the Standard Evaluation Systems of Rice (IRRI 2013). CTS scores at 7 (CTS7), 14 (CTS14) and 21 (CTS21) days of treatment were calculated using the average rating based on the two replicates. Chlorophyll content $(\mathrm{CC})$ values were taken using a Minolta SPAD-502 (Minolta Camera Co. Ltd., Tokyo, Japan) before treatment and at 7 and 14 days of treatment on 10 random seedlings per genotype. CC was taken three times near the base of the third or fourth leaf. The three values were averaged to obtain a value for each of the 10 seedlings. The reading value for wilting or dead seedlings was assumed to be zero. The percentages of chlorophyll content reduction at 7 and 14 days of treatment (\%RC7 and \%RC14 respectively) were calculated in reference to the measurement before the treatment.
Genotyping with SSR- and CG-based markers

The 18 genotypes were genotyped to estimate their genetic diversity at 32 SSR loci (Table 1, Supplementary file 1) linked to QTL for CT (Andaya and Mackill 2003a, b) and at six SSR- and CG-based markers: CT37-1, CT38-1, CT710F, CT720A, CT740C and CT780B (Andaya and Tai 2006). CT37-1 and CT38-1 flank the qCTS12 region, a major QTL for CTS, whereas CT710F, CT720A, CT740C and CT780B were designed to amplify regions from four openreading frames between CT37-1 and CT38-1 (Table 2, Supplementary file 1). All SSR- and CG-based markers used were obtained by mapping a population derived from the cultivars M-202 (cold-tolerant, japonica) and IR50 (cold-susceptible, indica). M-202 and IR50 were used hereby as controls for phenotyping and genotyping. Sequences of SSR

Table 2 Germination percentage (GP) of the 18 genotypes evaluated subjected to germination at $16^{\circ} \mathrm{C}$

\begin{tabular}{|c|c|c|c|}
\hline \multirow[t]{2}{*}{ Genotype } & \multicolumn{3}{|c|}{ Germination percentage $(\mathrm{GP})^{\mathrm{a}}$} \\
\hline & Day $5^{\mathrm{b}}$ & Day $7^{\mathrm{c}}$ & Day $14^{\mathrm{d}}$ \\
\hline Koshihikari & $0.0 \pm 0.0$ & $48.0 \pm 17.1$ & $92.0 \pm 2.0$ \\
\hline ECR61 & $0.0 \pm 0.0$ & $61.3 \pm 10.1$ & $82.0 \pm 9.2$ \\
\hline ECR67 & $0.0 \pm 0.0$ & $96.0 \pm 2.0$ & $100.0 \pm 0.0$ \\
\hline Ant11786 & $2.7 \pm 1.1$ & $90.0 \pm .4 .0$ & $99.3 \pm 1.2$ \\
\hline IR50 & $3.3 \pm 4.1$ & $30.7 \pm 5.8$ & $44.0 \pm 6.9$ \\
\hline Silewah & $4.7 \pm 3.1$ & $84.7 \pm 5.0$ & $96.7 \pm 2.3$ \\
\hline CR675 & $8.7 \pm 5.8$ & $84.7 \pm 5.0$ & $96.7 \pm 3.1$ \\
\hline Somewake & $14.0 \pm .7 .2$ & $91.3 \pm 5.0$ & $96.0 \pm 5.3$ \\
\hline IRGA 409 & $14.0 \pm 7.2$ & $93.3 \pm 3.1$ & $98.7 \pm 1.2$ \\
\hline ECR153 & $17.3 \pm 8.1$ & $96.0 \pm 0.0$ & $100.0 \pm 0.0$ \\
\hline M-202 & $18.0 \pm 2.0$ & $95.3 \pm 3.1$ & $97.3 \pm 1.2$ \\
\hline ECR57 & $18.0 \pm 2.0$ & $96.0 \pm 4.0$ & $100.0 \pm 0.0$ \\
\hline $\mathrm{H} 298 \mathrm{a} / 90$ & 31.3. \pm 7.0 & $91.3 \pm 6.1$ & $98.7 \pm 1.2$ \\
\hline Cambá INTA & $56.7 \pm 8.1$ & $95.3 \pm 5.0$ & $99.3 \pm 1.2$ \\
\hline El Paso 144 & $62.0 \pm 15.9$ & $96.7 \pm 3.1$ & $97.3 \pm 2.3$ \\
\hline L-202 & $63.3 \pm 11.0$ & $98.7 \pm 1.2$ & $99.3 \pm 1.2$ \\
\hline IRGA 417 & $64.7 \pm 4.2$ & $99.3 \pm 1.2$ & $100.0 \pm 0.0$ \\
\hline Puitá INTA CL & $73.3 \pm 7.0$ & $97.3 \pm 3.1$ & $98.0 \pm 2.0$ \\
\hline
\end{tabular}

${ }^{\mathrm{a}}$ Mean value and standard deviation of GP of four replicates of 50 seeds each

${ }^{\mathrm{b}, \mathrm{c}, \mathrm{d}} \mathrm{GP}$ at 4,7 and 14 days from the beginning of treatment, respectively 
primers were downloaded from the Gramene database (https://www.gramene.org), whereas sequences of SSR- and CG-based markers for qCTS12 were obtained from Andaya and Tai (2006).

DNA was obtained from $200 \mathrm{mg}$ of fresh leaf following the protocol described by Dellaporta (1983). PCR reactions for SSR consisted of $1 \mathrm{X}$ PCR buffer (750 mM Tris- $\mathrm{HCl} \mathrm{pH} 8.8,200 \mathrm{mM}(\mathrm{NH} 4)_{2} \mathrm{SO}_{4}$, $0.1 \%$ (v/v) Tween 20), $2.5 \mathrm{mM} \mathrm{MgCl}_{2}, 250 \mu \mathrm{M}$ of each primer, $250 \mu \mathrm{M}$ of each dNTP, $50 \mathrm{ng}$ template DNA and 0.8 units Taq polymerase (Fermentas, USA) in a final volume of $25 \mu \mathrm{l}$ in 96 -well plates. Amplifications were performed in a MyCycler thermocycler (Bio-Rad Laboratories, Inc.) with the following PCR profile: $95^{\circ} \mathrm{C}$ for $4 \mathrm{~min}, 35$ cycles of $95^{\circ} \mathrm{C}$ for $1 \mathrm{~min}$, $60 / 55^{\circ} \mathrm{C}$ for $1 \mathrm{~min}, 72{ }^{\circ} \mathrm{C}$ for $1 \mathrm{~min}$, and final extension of $72{ }^{\circ} \mathrm{C}$ for $7 \mathrm{~min}$. CG-based markers were assessed by PCR reactions with modifications: $300 \mu \mathrm{M}$ of each primer, $300 \mu \mathrm{M}$ of each dNTP, 200 ng DNA, 2 units Taq Polymerase and $55^{\circ} \mathrm{C}$ for $1 \mathrm{~min}$ for annealing. The PCR products of SSR were run on $1.5 \%$ agarose $/ 0.7 \%$ Synergel (Diversified Biotech, USA) or in 6\% acrylamide gel using MegaGel system (C.B.S. Scientific Company, Inc.) and visualized using ethidium bromide according to the manufacturer's recommended protocols. The PCR products of CG-based markers were run on $1.5 \%$ agarose and visualized by ethidium bromide.

Identification of contrasting genotypes through biplot clustering

The phenotyping and genotyping data from the 18 rice genotypes were analyzed using the Infostat software (Di Rienzo et al. 2011). Phenotypic variables from LTG and CTS screening were subjected to analysis of variance (ANOVA) after checking the assumption of normality (Supplementary files 3, 4 and 5). When normality was rejected, variables were analyzed after square root transformation. In all cases, mean values were compared by the D.G.C method to detect clusters (Di Rienzo et al. 2002). Principal Component Analysis (PCA) with standardized variables was performed to cluster tolerant/susceptible controls and breeding lines for LTG and CTS response. Molecular data were analyzed and PCR products were scored as present (1) or absent (0); the ratio between the total number of bands and the number of polymorphic bands and the polymorphic information content of each marker were calculated. A similarity distance matrix of the Dice coefficient was used for Principal Coordinate Analysis (PCooA) and, then, the conformations of PCA and PCooA were compared using a Generalized Procrustes Analysis. Contrasting genotypes for CT and with a maximum genetic distance from the marker loci linked to CT were selected as parents of bi-parental $F_{2: 3}$ segregating populations.

QTL mapping and analysis of CG-based markers in the segregating population

The selected parents, Puitá INTA CL and H298a/90, were genotyped with 110 additional markers (Supplementary file 2) to identify additional polymorphisms between them. Therefore, a population of $128 \mathrm{~F}_{2}$ plants was genotyped with 43 SSR- and CG-based markers and 15-25 seedlings from $89 \mathrm{~F}_{2}:_{3}$ derived families $(\sim 1800$ seedlings) were screened for CTS under $9{ }^{\circ} \mathrm{C}$ at 7 and 14 days of treatment (CTS7 and CTS14). QTL mapping was tested using single marker analysis. Additional $\mathrm{R}^{2}$ was used to express the percentage of the phenotypic variation explained by the QTL. Statistical analysis was done using QTL IciMapping v3.3 (Wang et al. 2012). The CG-based markers CT710F and CT720A were evaluated for their association with CTS in the $\mathrm{F}_{2: 3}$ families through ANOVA and mean comparison test by the D.G.C method. The genomic coordinates of markers and QTL were based on the annotation data on OsNipponbare-Reference-IRGSP-1.0 in the Gramene database (Tello-Ruiz et al. 2018).

\section{Results}

\section{LTG screening}

At low temperature, germination was slower and heterogeneous (Table 2). Differences in GP between genotypes were higher at 4 days of treatment, when this index ranged from 0 to $73.3 \%$, and decreased by 14 days of treatment, when only the susceptible genotype IR50 showed a low GP (44\%).

The variability between genotypes for LTG was detected with all germination indices used: GV and GR1, GR2, GR3 and GR4 (Supplementary file 3). However, the classification of the genotypes was different because each of them measures different 
characteristics of the germination process. For results and detailed analysis, see Supplementary file 4.

Under the treatment conditions and for all germination indices, the indica cultivars Cambá INTA, El Paso 144, IRGA 417, and Puitá INTA CL and the japonica cultivar L-202 showed the best performance for LTG. The susceptible control IR50 showed extremely low LTG owing to its low speed of germination and GP, whereas other genotypes selected as indica susceptible controls such as IRGA 409, El Paso 144 and IRGA 417 showed intermediate or high LTG. On the other hand, the tolerant control Koshihikari was classified as having low LTG owing to its delayed germination, although, unlike IR50, it reached high GP at 14 days.

At 14 days, when the treatment conditions were changed to $12 \mathrm{~h}$ light/12 h darkness, the genotypes developed between germination stages 07 and 09, with emerged coleoptile or first imperfect leaf emerging (Lancashire et al. 1991). Hereafter, susceptible genotypes showed stunted growth, slow chlorophyll synthesis in the plumule and/or seedling death before 28 days.

Considering all indices for early seedling growth, LTG variability was evident among genotypes (Supplementary file 3). Coleoptile growth (Supplementary file 5 Fig. 1a and 1b) was significantly higher for the tolerant controls L-202, M-202, Somewake and Silewah. The average radicle growth was significantly higher for M-202 and L-202 tolerant controls and the H298a/90 line, because the 18 genotypes showed different proportions of coleoptile and radicle growth.

According to the total seedling growth under low temperature, the best performing genotypes were the tolerant controls L-202 and M-202, followed by the tolerant controls Somewake, Silewah and H298a/90. For DWT/S, Puitá INTA CL was similar to the tolerant genotypes Somewake, Silewah and H298a/90 owing to its higher GV but with a smaller number of normal surviving seedlings per dish at 28 days.

The PCA for the germination stage (Fig. 1a) was performed using descriptive variables with lowest correlation values. PC1 and PC2 accounted for 70.3\% of the total variation. $\mathrm{PC} 1$ differentiated the susceptible controls IR50 and IRGA409, the breeding lines ECR61, CR675, ECR67 and the tolerant control Koshihikari towards negative values. Indeed, these genotypes showed lower speed of germination and delayed growth under low temperature. Interestingly, the tolerant control Koshihikari and ECR67 exhibited dark green coleoptiles as a sign of tolerance. On the other hand, PC1 grouped the tolerant genotypes towards positive values, whereas PC2 differentiated two groups on this plane. Puitá INTA CL, Cambá INTA and the susceptible controls El Paso 144 and IRGA 417 were on the upper right plane of the biplot and showed the highest LTG according to the values of germination indices. These genotypes exhibited susceptibility when the conditions were changed to $12 \mathrm{~h}$ light/ $12 \mathrm{~h}$ darkness. This was evidenced by stunted growth and chlorosis according to DWT, DWT/S and CT. Towards the lower right plane, PC2 grouped the tolerant controls L-202, M-202, Somewake and Silewah, and the Argentine breeding line H298a/90. These genotypes showed the lowest values of germination indices, although they had tolerance towards the end of the germination stage. This tolerance was evidenced by greater normal growth seedling (DWT, DWR/S $\% \mathrm{~S}>0.5$ and $\% \mathrm{NS}$ ) and lower CT values due to chlorophyll synthesis. L-202 showed high germination ability and later growth towards the end of the germination stage under the low temperature (Supplementary files 4 and 5).

Cold tolerance at the seedling stage (CTS)

Variability for \% RC7 and \%RC14 was evident among genotypes $\left(\mathrm{R}^{2}=0.70, p<0.0001, \mathrm{CV}\right.$ : 37.90; $\mathrm{R}^{2}=0.86, p<0.0001, \mathrm{CV}: 19.98$, respectively). The D.G.C. test grouped the tolerant genotypes with lower values, such as $\mathrm{H} 298 \mathrm{a} / 90$ and the controls M-202, L-202, Somewake and Silewah, and the susceptible genotypes, including Puitá INTA CL and the controls IRGA409, El Paso 144, IRGA417, and IR50, with higher values (Table 3). At 21 days of treatment, the extensive leaf rolling and seedling mortality in the sensitive genotypes made it impossible to measure chlorophyll content. The CTS7 and CTS14 values of visual scoring were significantly correlated with \%RC7 and \%RC14 (Spearman's $\mathrm{r}=0.81-0.97$ ), thus indicating that the subjective CT scale used was effective to detect cold-temperature-induced chlorosis.

The PCA biplot (Fig. 1b) showed that PC1 and PC2 accounted for $94 \%$ of the total variation. PC1 differentiated two contrasting groups for CT. The japonica tolerant controls M-202, Silewah, Somewake, L-202, Koshihikari and the line H298a/90 (originated from a 


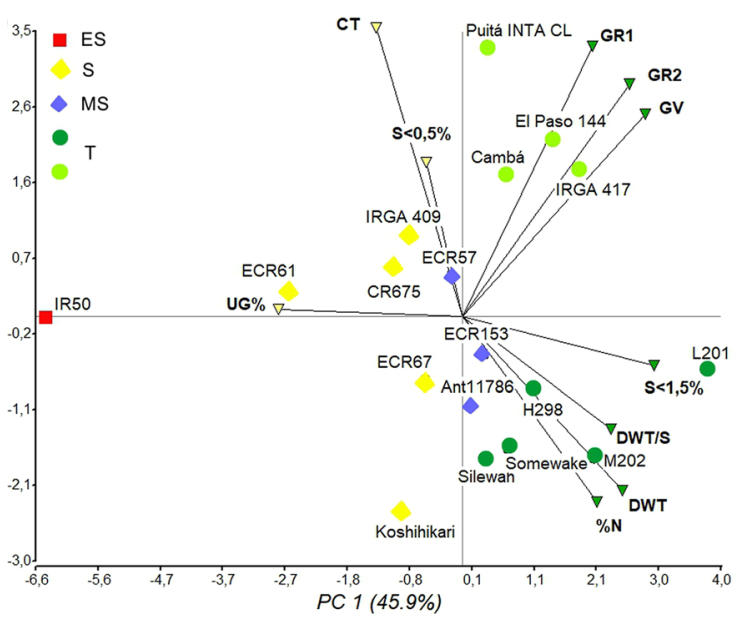

Fig. 1 Plot of PC1 and PC2 from indices for the evaluation and classification of 18 rice genotypes at germination (LTG) and vegetative stage (CTS): $E S$ extremely susceptible; $S$ susceptible; $M S$ moderately susceptibility; $T$ tolerant. a LTG scoring at $16{ }^{\circ} \mathrm{C}$ using indices for evaluation during the first 14 days in darkness and during early seedling growth under $12 \mathrm{~h}$ light/12 h darkness to complete 28 days. GV: germination vigor; GR1 and GR3: germination rate; DWT: total dry weight of seedlings; DWT/S: average of total dry weight for normal seedlings; \%US:

japonicalindica cross) clustered on the left quadrants with the lowest \% RC and CT score (Table 3) and with little visual damage at 21 days, although with delayed growth (Fig. 3). On the other hand, the indica susceptible controls IR50, IRGA 417, IRGA 409, El Paso 144 and Puitá INTA CL clustered towards the right quadrants. IR50, extremely susceptible, showed an early and large \% RC, whereas other susceptible genotypes, exhibited a delay in the occurrence of visual damage and seedling death. The Argentine breeding lines near the center of the graph showed intermediate CTS.

Selection of contrasting genotypes for QTL mapping

Twenty-nine of the SSR- and CG-based markers linked to CTS were used to genotype the mini-core set of 18 rice genotypes. The values of polymorphic information content ranged between 0.20 and 0.83 , and $90.91 \%$ of the 110 total bands were polymorphic. PCooA (Fig. 2a) showed that the indica susceptible cultivars IR50, IRGA 417, IRGA 409, El Paso 144 and Puitá INTA CL were tightly grouped by an average

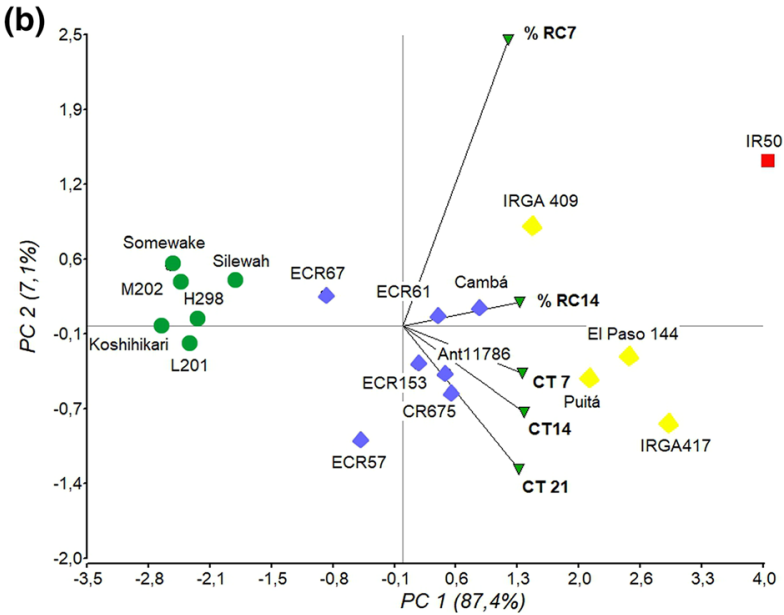

percentage of unterminated seeds; $\% \mathrm{~S}<0.5$ and $\% \mathrm{~S}>0.5$ : seedlings with shoots both smaller and larger than $0.5 \mathrm{~cm} ; \% \mathrm{NS}$ seedlings with normal development. $C T$ cold tolerance evaluated with a visual scale (1 tolerant to 9 susceptible). B CTS evaluation on seedlings at the 3-4 leaf stage subjected to $9{ }^{\circ} \mathrm{C}$ under $12 \mathrm{~h}$ light/12 h darkness for 21 days. \%RC7 and \%RC14: reduction of chlorophyll content at 7 and 14 days; CTS7, CTS14 and CTS21: visual scale at 7,14 and 21 days

similarity of 0.81 . The genotypes with intermediate susceptibility, Cambá INTA, Ant11786, ECR61, CR675, ECR 57 and ECR153, were closer to the indica genotypes, with an average similarity of 0.69 . These susceptible genotypes with CTS scores of 5, 7 and 9 showed the same PCR products as IR50 for the CG-based markers (Table 4). On the other hand, the japonica tolerant cultivars M-202, Silewah, L-202, Somewake, Koshihikari and the line H298a/90 formed a distant group with a range of similarity from 0.45 to 0.88 among them and with an average similarity of 0.29 with respect to the indica group. The long-grain line $\mathrm{H} 298 \mathrm{a} / 90$ showed higher genetic similarity to M-202 (0.88), a medium-grain japonica cultivar (Fig. 2a). All tolerant genotypes with CTS scores at 21 days of 1 and 3 showed the same marker allele for CG-based markers as M-202 (Table 4).

The classification from phenotyping for CTS (PCA) and the genotyping using molecular data (PCooA) presented $86.4 \%$ of consensus. According to the Generalized Procrustes Analysis (Fig. 2b), PC1 accounted for $90 \%$ of the variation, and the tolerant genotypes, which clustered at PC1 quadrants, lacked cold stress symptoms at 21 days (Fig. 3c). 
Table 3 Evaluation of cold tolerance at seedling stage (CTS at 3-4 leaf stage) at $9{ }^{\circ} \mathrm{C}$ (constant temperature) for 21 days

\begin{tabular}{|c|c|c|c|c|c|c|c|c|}
\hline Genotype & $\% \mathrm{RC}^{3}$ & & $\mathrm{CT} 7^{4}$ & Genotype & $\% \mathrm{RC} 14^{3}$ & & $\mathrm{CT} 14^{4}$ & $\mathrm{CT} 21^{4}$ \\
\hline Koshihikari $^{1}$ & 5.11 & a & 1 & $\mathrm{M}-202^{1}$ & 16.59 & $\mathrm{a}$ & 1 & 1 \\
\hline L-202 ${ }^{1}$ & 7.38 & $\mathrm{a}$ & 1 & H298a/90 & 21.93 & $\mathrm{a}$ & 1 & 3 \\
\hline Somewake $^{1}$ & 11.66 & $\mathrm{a}$ & 1 & L-202 ${ }^{1}$ & 23.32 & a & 1 & 3 \\
\hline $\mathrm{H} 298 \mathrm{a} / 90$ & 11.71 & $\mathrm{a}$ & 1 & Koshihikari $^{1}$ & 25.47 & a & 1 & 1 \\
\hline ECR57 & 11.82 & $\mathrm{a}$ & 3 & Somewake $^{1}$ & 27.61 & $\mathrm{a}$ & 1 & 1 \\
\hline M-202 ${ }^{1}$ & 15.03 & $\mathrm{a}$ & 1 & ECR57 & 28.97 & $\mathrm{a}$ & 5 & 7 \\
\hline Silewah ${ }^{1}$ & 17.09 & a & 1 & Silewah $^{1}$ & 37.58 & $\mathrm{~b}$ & 1 & 3 \\
\hline ECR67 & 20.13 & $\mathrm{~b}$ & 3 & Ant11786 & 41.39 & $\mathrm{~b}$ & 5 & 7 \\
\hline CR675 & 20.9 & $\mathrm{~b}$ & 5 & Cambá INTA & 47.02 & $\mathrm{~b}$ & 5 & 7 \\
\hline ECR153 & 23.02 & $\mathrm{~b}$ & 3 & ECR67 & 48.11 & $\mathrm{~b}$ & 3 & 5 \\
\hline Ant11786 & 24.4 & $\mathrm{~b}$ & 5 & ECR153 & 48.88 & $\mathrm{~b}$ & 5 & 7 \\
\hline IRGA417² & 28.22 & $\mathrm{~b}$ & 7 & ECR61 & 49.95 & $\mathrm{~b}$ & 5 & 7 \\
\hline El Paso $144^{2}$ & 29.65 & $\mathrm{~b}$ & 5 & CR675 & 50.29 & $\mathrm{~b}$ & 5 & 7 \\
\hline ECR61 & 30.8 & $\mathrm{~b}$ & 3 & ${\text { IRGA } 409^{2}}^{2}$ & 64.08 & $\mathrm{c}$ & 5 & 7 \\
\hline Cambá INTA & 34.46 & $\mathrm{~b}$ & 5 & Puitá INTA CL & 80.04 & $\mathrm{~d}$ & 7 & 9 \\
\hline Puitá INTA CL & 36.66 & $\mathrm{~b}$ & 7 & El Paso $144^{2}$ & 89.43 & $\mathrm{~d}$ & 7 & 9 \\
\hline IRGA $409^{2}$ & 46.91 & $\mathrm{c}$ & 5 & IRGA417² & 100 & $\mathrm{e}$ & 9 & 9 \\
\hline $\operatorname{IR} 50^{2}$ & 69.98 & $\mathrm{~d}$ & 7 & $\operatorname{IR} 50^{2}$ & 100 & $\mathrm{e}$ & 9 & 9 \\
\hline
\end{tabular}

${ }^{1}$ Cold tolerant control at seedling stage

${ }^{2}$ Cold susceptible control at seedling stage

${ }^{3}$ Percent reduction of chlorophyll content at 7 (\%RC7) and $14(\% \mathrm{RC} 14)$ days. Different letters indicate significant differences $(\alpha=0.05)$ for D.G.C mean comparison test (Di Rienzo et al. 2002)

${ }^{4}$ Visual scoring of cold tolerance (CT) at 7 (CT7), 14 (CT14), and 21 (CT21) days according to the Standard Evaluation Systems of rice (IRRI 2013): 1 = seedlings dark green, 3 = seedlings light green, $5=$ seedlings yellow, $7=$ seedlings brown, $9=$ seedlings dead

Furthermore, two groups of susceptible genotypes that clustered together at the molecular level showed severe symptoms and seedling death (Fig. 3a, b). Thus, the local H298a/90 (tolerant) and Puitá INTA CL (susceptible) parents were selected as contrasting genotypes at both phenotypic and molecular level for QTL mapping.

QTL mapping and analysis of CG-based markers in the segregating population

The screening of 159 SSR- and CG-based markers allowed the detection of 70 polymorphic loci (44\%) between H298a/90 and Puitá INTA CL and 43 of these markers were finally useful to genotype the bi-parental segregating population (Supplementary file 2). Because of the flower sterility of $F_{2}$ plants and poor germination of $\mathrm{F}_{3}$ seeds, only $89 \mathrm{~F}_{2}:_{3}$ families were finally obtained to be evaluated at 7 and 14 days under low temperature. Under these conditions, the seedlings obtained exhibited all the range of scores from 1-9 (Table 5). The distribution for CTS7 was normal (Shapiro Wilks test $\mathrm{W}=0,969$ and $p=0.1598$ ), whereas that for CTS14 was near normal $(\mathrm{W}=0.959$, $p=0.0284$ ), a result that supports mapping analysis (Broman and Sen 2009). Despite the low number of markers used for QTL mapping using single marker analysis, two major QTL, $q$ CTS11 and $q$ CTS12, were detected in chromosomes 11 and 12, respectively. These two QTL were called $q C T S$ according to the known QTL retrieved from the Gramene database (Table 6). qCTS11, linked to RM144 in chromosome 11 (11:28,804,933-28,805,157), accounted for $18.49 \%$ of the phenotypic variance and was detectable only after 7 days of treatment (CTS7). In addition, $q C T S 12$, at chromosome 12 , was associated with CTS at both 7 and 14 days of treatment (CTS7 and CTS14) and was linked to the markers RM247, 


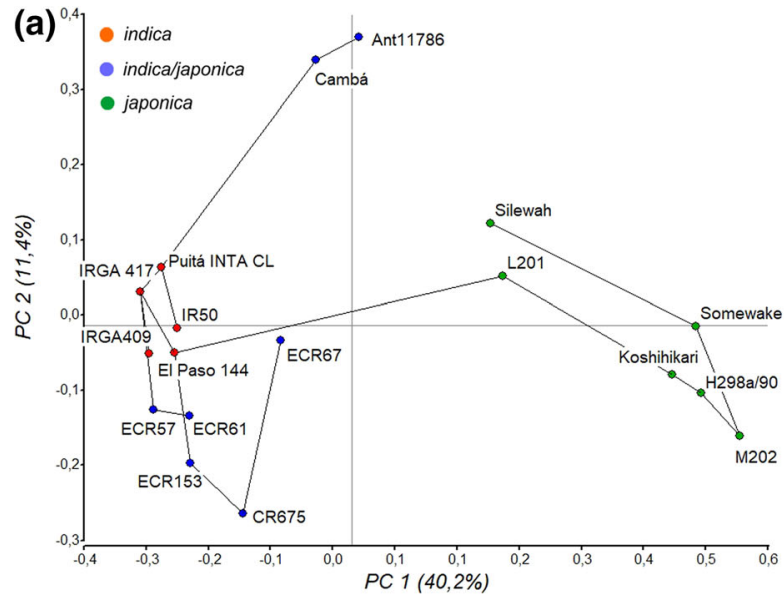

Fig. 2 a Plot of PC1 v. PC2 from PCooA of genotyping data with minimum spanning trees (MST) of 18 rice genotypes genotyped with SSR- and GC-based markers linked to CTS. b Plot of PC1 v. PC2 from generalized procrustes analysis with MST resulting from the comparison of PCA obtained from

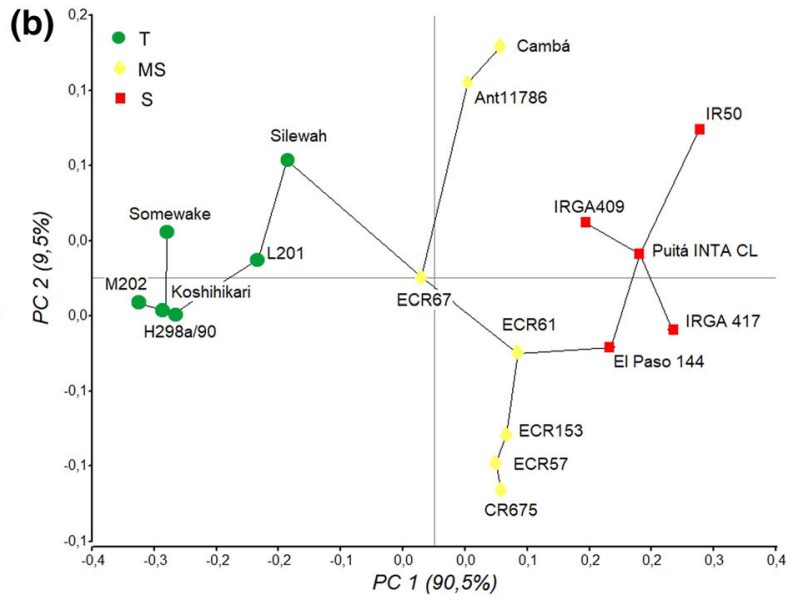

phenotyping of CTS and PCooA. According to the results of the generalized procrustes analysis, the genotypes evaluated were finally classified as $S$ susceptible; $M S$ moderately susceptibility; $T$ tolerant
Table 4 PCR products (bp) of candidate gene-markers on the major QTL $q C T S 12$ corresponding to CT710F, CT720A, CT740, CT780 and CTS visual score values of seedlings at $9^{\circ} \mathrm{C}$ for 21 days

*Size of the CG-based marker for M-202 (coldtolerant control)

**Size of CG-based marker for IR50 (cold-susceptible control)

${ }^{1}$ Cold tolerant control at seedling stage

${ }^{2}$ Cold susceptible control at seedling stage

\begin{tabular}{llllrl}
\hline Genotype & CT710F & CT720A & CT740 & CT780 & CTS21 \\
\hline M-202 ${ }^{1}$ & $1000^{*}$ & $1000^{*}$ & $900^{*}$ & $760^{*}$ & 1 \\
IR50 $^{2}$ & $950^{* *}$ & $1150^{* *}$ & - & $780^{* *}$ & 9 \\
CR675 & 950 & 1150 & - & 780 & 7 \\
ECR153 & 950 & 1150 & - & 780 & 7 \\
ECR57 & 950 & 1150 & - & 780 & 7 \\
ECR67 & 950 & 1150 & - & 780 & 5 \\
ECR61 & 950 & 1150 & - & 780 & 7 \\
EL Paso 144 & 950 & 1150 & - & 780 & 9 \\
IRGA 409 & 950 & 1150 & - & 780 & 7 \\
IRGA417 & 950 & 1150 & - & 780 & 9 \\
Puitá INTA CL & 950 & 1150 & - & 780 & 9 \\
H298a/90 & 1000 & 1000 & 900 & 780 & 3 \\
Koshihikari & 1000 & 1000 & 900 & 760 & 1 \\
L-202 & 1000 & 1000 & 900 & 760 & 3 \\
Silewah & 1000 & 1000 & 900 & 760 & 3 \\
Somewake & 1000 & 1000 & - & 760 & 1 \\
\hline
\end{tabular}

CT710 and CT720 (Table 6). The SSR RM519 showed association only with $q$ CTS12 at 14 days (CTS14), when severe damage was evident. The CGbased markers CT710 and CT720 explained a high proportion of the phenotypic variability at 7 (25.15\%) and 14 days (46.51\%). Finally, the average CTS7 and CTS14 scores of the $\mathrm{F}_{2}: 3$ families derived from homozygous $\mathrm{F}_{2}$ plants that carried the tolerant alleles at CG-based markers from CT710F and CT720A were significantly lower than those of families derived from heterozygous or homozygous $\mathrm{F}_{2}$ plants with susceptible alleles at CG-based markers (Fig. 4).

\section{Discussion}

The rice phenological germination stage includes the germination process and early seedling growth until 
(a)

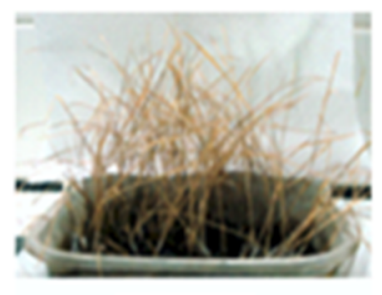

IR50

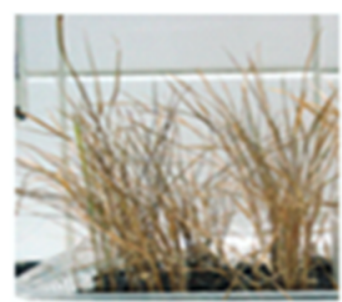

Puitá INTA CL

\section{CTS21 $=9$}

(b)
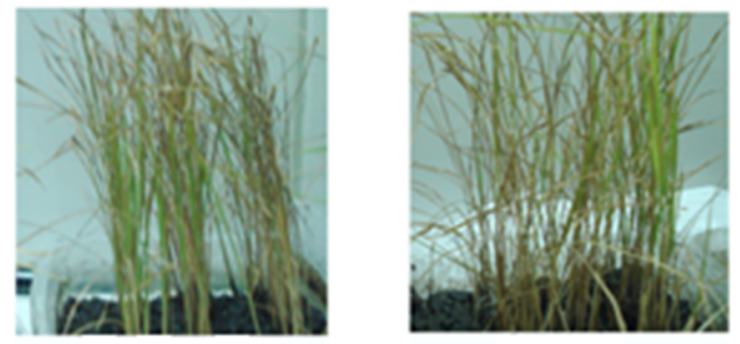

IRGA406

\section{CTS21=7}

CR675

(c)
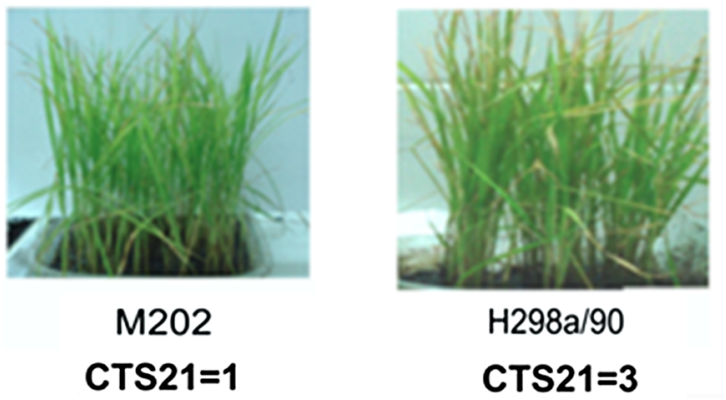

$\mathrm{H} 298 \mathrm{a} / 90$

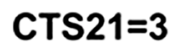

Fig. 3 Cold tolerance at seedling stage (CTS) of the 18 rice genotypes evaluated at $9{ }^{\circ} \mathrm{C}$ for 21 days using CT visual scoring. a The susceptible control IR50 and Puitá INTA CL were classified as CTS21 $=9$ (dead seedlings). b The susceptible control IRGA 409 and the Argentine breeding line CR675 were classified as CTS21 = 7 (brown seedlings). $\mathbf{c}$ The tolerant control M-202 was classified as CTS21 $=1$ (dark green seedlings) and the Argentine breeding line H298a/90 was classified as CTS21 = 3 (dark green seedlings with brown leaf tips)

the emergence of the first imperfect leaf (Lancashire et al. 1991). To evaluate germination or early seedling growth under temperatures between 13 and $17{ }^{\circ} \mathrm{C}$, researchers have used different indices. These indices are often time-consuming because they require daily or laborious evaluations, such as the assessment of the reduction of coleoptile length and DW under low temperature in relation to a control genotype (Cruz and Milach 2004; Ye et al. 2009; Satoh et al. 2016).

The method used in this study described the LTG of 18 indica, japonica and mixed genotypes from indica and japonica genetic background by using simple scores for both germination and early seedling growth that simulate the response in field conditions at suboptimal temperature during the rice sowing season. The three germination counts performed to calculate germination indices were sufficient to describe the heterogeneity and the reduction in the germination due to low temperature. Counts made at 7 days between two extreme moments discriminated genotypes with intermediate behavior from those with higher germinability and precocity (4 days) or delayed germination (14 days) (Ranal and Santana 2006).

In this study, the germinability of all genotypes, especially that of the indica susceptible control IR50, was affected by low temperature. Cultivars with indica background such as IRGA 417, El Paso144, Puitá INTA CL and Cambá INTA showed the highest germinability even at low temperature. At optimal temperature $\left(28{ }^{\circ} \mathrm{C}\right)$, they reached $100 \%$ germination at 4 days, but, at $16{ }^{\circ} \mathrm{C}$, they were affected mostly in the speed of germination and reached more than $97 \%$ of GP at 14 days. Even so, they were the earliest to germinate and showed greater initial growth. However, when exposed to light (between 14 and 28 days), they showed stunted growth and chlorosis. At 28 days under low temperature, the growth of these cultivars was similar to or lower than that of the japonica tolerant controls M-202, Somewake and Silewah and the tolerant breeding line H298a/90. These genotypes, with intermediate or low germinability, were able to synthesize chlorophyll (greening phenotype) and sustain growth under the interaction of low temperature and light. Thus, germinability (i.e. germination indices) and early growth (i.e. coleoptile size or total DW) were the two non-correlated traits that best explained LTG into subcomponents.

Apart from DW, the number of normal and larger seedlings (i.e. $\% \mathrm{NS}$ and $\% \mathrm{~S}>0.5$ ) and the CT score (lower values) have been useful indices to assess seedling survival without chlorosis or wilting phenotype due to the light (Sthapit and Witcombe, 199, Mahender et al. 2015). For example, IRGA 417 and Koshihikari have been found to be tolerant at germination stage at $13{ }^{\circ} \mathrm{C}$ for 28 days in darkness (Cruz 
Table 5 Distribution of means for cold tolerance at seedling stage (CTS) of Puitá INTA CL, H-298a/90 and 89 F $2: 3$ families

\begin{tabular}{|c|c|c|c|c|c|c|c|c|}
\hline \multirow[t]{2}{*}{ Trait } & \multicolumn{2}{|l|}{ Parents $^{1}$} & \multicolumn{6}{|c|}{$\mathrm{F}_{2}:{ }_{3}$ Families $^{2}$} \\
\hline & Puitá INTA CL & H298a/90 & Mean & Median & Min-Max & S.D & Skewness & Kurtosis \\
\hline CTS7* & $7.75 \pm 1.04$ & $2.00 \pm 1.07$ & 4.97 & 4.82 & $2.4-7.5$ & 1.10 & 0.089 & -0.805 \\
\hline CTS14* & $8.50 \pm 0.93$ & $2.50 \pm 0.93$ & 6.48 & 6.52 & $3.40-9.0$ & 1.43 & -0.127 & -0.852 \\
\hline
\end{tabular}

*Means of CTS7 and CTS14 of Puitá INTA CL and H298a/90 are significantly different, with $p<0.05$ for the $t$ test

${ }^{1}$ Parental means were obtained from 10 seedlings of each genotype used as test control

${ }^{2}$ Means for each $\mathrm{F}_{2: 3}$ family $(\mathrm{n}=89$ ) were obtained by evaluating $15-25$ seedlings for each family

Table 6 QTL detected for cold tolerance at seedling stage (CTS). Seedlings of a $\mathrm{F}_{2: 3}$ population derived from Puitá INTA CL (cold susceptible) and an Argentine breeding line H298a/90 (cold tolerant) were grown at $9{ }^{\circ} \mathrm{C}$ (constant temperature) and evaluated at 7 (CTS7) and 14 (CTS14) days

\begin{tabular}{lllclll}
\hline Trait $^{1}$ & Chr. $^{2}$ & Marker & LOD $^{3}$ & $\mathrm{R}^{2}(\%)^{4}$ & Ef. Ad $^{5}$ & Correspondence $^{6}$ \\
\hline CTS7 & 11 & RM144 & 3.95 & 18.49 & 0.6067 & qCTS11-2; ${ }^{2}=13$ \\
& & & & & qCTP11 R $\mathrm{R}^{2}=22.2$ \\
& 12 & RM247 & 3.05 & 14.59 & 0.3729 & qCTS12a; R $=40.6$ \\
& 12 & CT710 & 5.59 & 25.15 & 0.7163 & qCTP12 R ${ }^{2}=27.2$ \\
CTS14 & 12 & CT720 & 5.59 & 25.15 & 0.7163 & \\
& 12 & RM247 & 6.49 & 28.56 & 0.8581 & \\
& 12 & CT710 & 12.09 & 46.51 & 1.3284 & \\
& 12 & CT720 & 12.09 & 46.51 & 1.3284 & \\
& 12 & RM519 & 4.09 & 19.07 & 0.8598 & \\
\hline
\end{tabular}

${ }^{1}$ Seedlings of an $\mathrm{F}_{2: 3}$ population derived from Puitá INTA CL (cold susceptible) and an Argentine breeding line H298a/90 (cold tolerant) were grown at $9{ }^{\circ} \mathrm{C}$ (constant temperature) and evaluated at 7 (CTS7) and 14 (CTS14) days

${ }^{2}$ chromosome position

${ }^{3}$ LOD score

${ }^{4}$ coefficient of determination $\left(\mathrm{R}^{2}\right)$

5 an additive positive effect indicates the contribution of Puitá INTA CL to cold susceptibility

${ }^{6}$ putative correspondence with previously reported QTL for cold tolerance at seedling stage ( $q C T S$ ) (Andaya and Mackill 2003a; Andaya and Tai 2006) and previously reported QTL for cold tolerance at plumule stage ( $q C T P$ ) (Baruah et al. 2009)

and Milach 2004). In our experiment, Koshihikari showed lower precocity for germination than IRGA417, but with similar values of DW, because it accumulated more biomass by photosynthesis, and showed higher \%NS. and no seedling mortality. The genotypes with high germinability under low temperature (e.g. Puitá INTA CL, IRGA 417) could be considered tolerant according to coleoptile/plumule growth only because of early vigor. At 28 days, they express susceptibility because low temperature inhibits chloroplast development and the exposure to light produces oxidative stress, leading to seedling death at the end of the germination stage (Yoshida et al. 1996; Zhang et al. 2014). Thus, phenotyping for LTG was successful by integrating germination indices and early seedling growth and multivariate analysis.

The comparison of PCA plots for LTG and CTS phenotyping showed that the genotypes with the ability to synthesize chlorophyll and survive under low temperature at the germination stage were tolerant at vegetative stages (3-4 leaves) under $9{ }^{\circ} \mathrm{C}$. These genotypes displayed lower reduction of chlorophyll content, necrosis and wilting. 
(a)

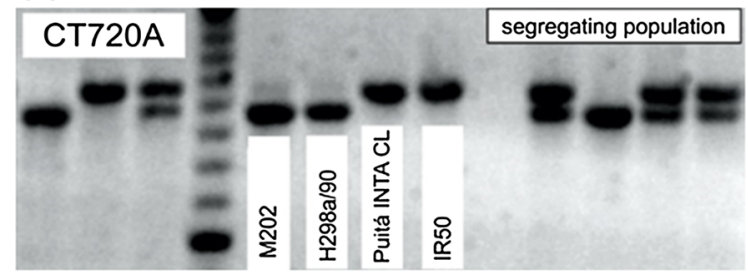

(b)

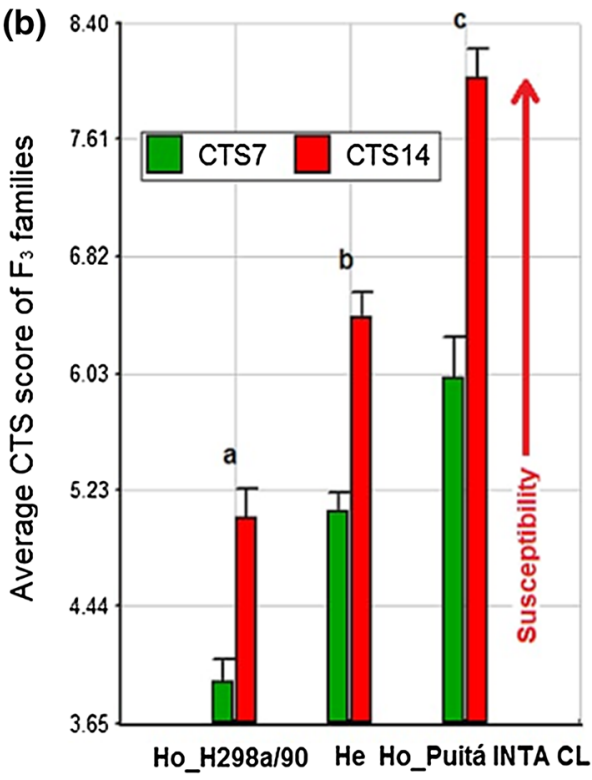

Allelic state of CG-based markers in $F_{2}$

Fig. 4 Analysis of CG-based markers linked to CTS in the segregating population derived from the parents H298a/90 (tolerant) and Puitá INTA CL (susceptible). a PCR products of marker CT720A corresponding to OsGSTZ1 in genotypes M-202 (tolerant control), H298a/90, IR50 (susceptible control), Puitá INTA CL and $\mathrm{F}_{2}$ plants. b The average visual score of CTS of $\mathrm{F}_{2: 3}$ families derived from homozygous and heterozygous $\mathrm{F}_{2}$ plants for parental alleles of CG-based markers. Visual scores were derived from the evaluation at 7 (CTS7) and 14 days (CTS14). X axis: allelic state of CT710A and CT720F of $\mathrm{F}_{2}$ plants: Ho_H298a/90 = homozygous for alleles of H298a/90; He = heterozygous; Ho_Puitá INTA CL = homozygous for alleles of Puitá INTA CL. Different letters indicate significant differences $(p<0.05)$ for D.G.C mean comparison test (Di Rienzo et al. 2002)

According to our results, tolerant controls (Somewake, Silewah, Koshihikari, L-202) and the Argentine breeding line H298a/90 may be able to adapt to prolonged low temperature from the end of the germination stage, similar to M-202 (Kim et al. 2011). Thus, H298a/90 may provide a useful source of genes to improve the widespread indica genotypes such as Puitá INTA CL, which, despite its greater germinability, exhibits susceptibility at later stages under suboptimal temperatures.

Through mapping studies, researchers have reported many QTL linked to SSR- and CG-based markers for CT; however, these QTL and markers have not been adequately exploited in breeding programs (Crossa et al. 2017). In crop breeding, researchers earlier assumed that markers associated with QTL from preliminary mapping studies were directly useful in marker-assisted selection (MAS) (Collard and Mackill 2008). The validation of QTL and markers for MAS must meet some requirements. Indeed, markers and QTL must keep linkage in different populations and the QTL has to be effective in different genetic backgrounds and environments (Bernardo 2008). The present study was successful in validating major QTL/genes for CTS associated with closely linked markers and provides an affordable means of incorporating MAS into breeding programs without the need of high-throughput phenotyping and genotyping facilities.

The QTL analysis of CT and fine-mapping from a cross between IR50 and M-202 provided useful SSRand CG-based markers linked to CTS (Andaya and Mackill 2003a, b; Andaya and Tai 2006). These markers were used to check for the correspondence of allelic variation of 18 rice genotypes and their variability for CTS. The agreement obtained by the Generalized Procrustes Analysis (86.4\%) between the genotyping and the phenotyping data showed high consistency. This result suggests that the selected markers, which had been previously linked to CT (Supplementary file 1), are associated with CTS in the 18 rice genotypes here evaluated. Particularly, CGbased markers for the major QTL qCTS12, CT720A, and $\mathrm{CT} 710 \mathrm{~F}$ discriminated all the tolerant genotypes from those with intermediate to high susceptibility to low temperature and may explain the contrast of CTS between the tolerant breeding line H298a/90 and the susceptible cultivar Puitá INTA CL.

In this study, by means of single marker analysis of an $\mathrm{F}_{2: 3}$ population derived from $\mathrm{H} 298 \mathrm{a} / 90$ and Puitá INTA CL and a few well-selected markers, we detected two major QTL for CTS on chromosomes 11 and 12 ( $q$ CTS11 and $q$ CTS12), in agreement with that reported by Andaya and Mackill (2003a). Other studies reported QTL on chromosomes 11 and 12 for CT. The RM144 marker on the long arm of 
chromosome 11, linked to $q$ CTS 11 in this study, is also linked to qCTGERM11-5 (11:19,639,219-22,014,679) (Borjas et al. 2016), qCTP11 (11:21,702,10022,480,832) (Baruah et al. 2009) and qCTS11-2 $(11: 22,480,808-28,805,157)$ (Andaya and Mackill 2003a). Wang et al. (2018) suggested that this location on chromosome 11 might be a hot spot for genes responsible for $\mathrm{CT}$ at the germination and seedling stages. Among them, a heat-shock protein 70 (LOC_Os11g47760) (Bonnecarrère et al. 2015) and a peptidase (LOC_Os11g33330) (Shakiba et al. 2017) have been proposed as candidate genes.

The genomic region between the genomic coordinates $3,186,473$ and $19,932,320$ on chromosome 12 is linked to markers RM247, RM519, CT720A and CT710F. In this region, $q C T P 12$ has been reported for cold tolerance at the plumule stage (Baruah et al. 2009), $q C T S 12 a$ and $q C T S 12 b$ for CTS, and $q C T S 12$ for CT at booting stage (Andaya and Mackill 2003a, b). These QTL are present in populations derived from crosses between ssp. japonica, ssp. indica and the wild rice Oryza rufipogon and may be related to the adaptation of rice to temperate or tropical regions (Zhang et al. 2005; Andaya and Tai 2006; Shakiba et al. 2017).

The marker CT710F targets the locus LOC_Os12g10710, which encodes a protein containing the NB-LRR domain for a response to biotic and abiotic stress (Van Ooijen et al. 2008). In addition, the marker CT720A targets the locus LOC_Os12g10720, which is adjacent to LOC_Os12g10730. These loci correspond to OsGSTZ1 and OsGSTZ2, respectively, and encode two glutathione S-transferases related to the tolerance to abiotic stresses such as cold, salt and drought by scavenging of reactive oxygen species (Andaya and Tai 2006). Kim et al. (2011) identified a single nucleotide polymorphism (SNP) in OsGSTZ2 (c.295A > G), which strongly correlated with CTS in 76 indica and japonica rice varieties. Moreover, Pachecoy et al. (2014) reported the same SNP correlated with CTS in 116 rice inbred lines and varieties from South America. These researchers analyzed several genotypes such as the susceptible cultivars IR50, IRGA417, IRGA409 (spp. indica, G allele) and the tolerant cultivars Somewake, Silewah and M-202 (spp. japonica, A allele). In our study, these cultivars were used as susceptible and tolerant controls. The CT710F and CT720A alleles are linked to the susceptibility/tolerance within the mini-core set of 18 genotypes (Table 4) and the segregating population derived from H298a/90 and Puitá INTA CL (Table 6). Thus, these markers are likely linked to the SNP c.295A > G in these genotypes.

Functional markers need to be developed to analyze the allelic variation at OsGSTZ2 and thus determine which OsGSTZ2 alleles are present to improve CTS in Argentine breeding populations. However, in this study, we identified three easily scored markers, RM144 (qCTS11), CT710F ( $q$ CTS12), and CT720A ( $q$ CTS12), which are linked to major QTL for CTS. These markers may therefore be used for introgression of favorable QTL alleles for CTS into genotypes with high LTG such as Puitá INTA CL to obtain breeding lines with early seedling vigor and well adapted to the sowing season in Argentina. Moreover, due to the simple discrimination of their PCR allele products on agarose and codominant segregation, these markers are useful to screen large populations in breeding programs without the need to conduct high throughput genotyping.

Acknowledgments We thank Dr. Sofía Eugenia Olmos and Dr. Julia Verónica Sabio Garcia for their useful comments and suggestions to improve the manuscript. Financial support from the National Institute of Agricultural Technology of Argentina (INTA) (Grant No. PNBIO1131042) (Research Grant \# AEBIO 241311 and Graduate Scholarship for M.L. Bonell) is acknowledged.

\section{References}

Andaya VC, Mackill DJ (2003a) Mapping of QTLs associated with cold tolerance during the vegetative stage in rice. J Exp Bot 54:2579-2585. https://doi.org/10.1093/jxb/ erg243

Andaya V, Mackill D (2003b) QTLs conferring cold tolerance at the booting stage of rice using recombinant inbred lines from a japonica $\times$ indica cross. Theor Appl Genet 106:1084-1090. https://doi.org/10.1007/s00122-0021126-7

Andaya VC, Tai TH (2006) Fine mapping of the qCTS12 locus, a major QTL for seedling cold tolerance in rice. Theor Appl Genet 113:467-475. https://doi.org/10.1007/s00122-0060311-5

Baruah AR, Ishigo-Oka N, Adachi M et al (2009) Cold tolerance at the early growth stage in wild and cultivated rice. Euphytica 165:459-470. https://doi.org/10.1007/s10681008-9753-y

Bernardo R (2008) Molecular markers and selection for complex traits in plants: learning from the last 20 years. Crop Sci 48:1649. https://doi.org/10.2135/cropsci2008.03.0131 
Bertin P, Kinet J, Bouharmont J (1996) Evaluation of chilling sensitivity in different rice varieties. Relationship between screening procedures applied during germination and vegetative growth. Euphytica 89:201-210. https://doi.org/ 10.1093/jxb/erg243

Bonnecarrère V, Quero G, Monteverde E et al (2015) Candidate gene markers associated with cold tolerance in vegetative stage of rice (Oryza sativa L.). Euphytica 203:385-398. https://doi.org/10.1007/s10681-014-1290-2

Broman K.W., Sen Ś. (2009) Non-normal phenotypes. In: A guide to QTL mapping with R/qtl. Statistics for biology and health. Springer, New York, NY

Collard BCY, Mackill DJ (2008) Marker-assisted selection: an approach for precision plant breeding in the twenty-first century. Philos Trans R Soc B Biol Sci 363:557-572. https://doi.org/10.1098/rstb.2007.2170

Collard BCY, Vera Cruz CM, McNally KL, et al (2008) Rice molecular breeding laboratories in the genomics era: Current status and future considerations. Int J Plant Genom 2008

Crossa J, Pérez-Rodríguez P, Cuevas J et al (2017) Genomic selection in plant breeding: methods, models, and perspectives. Trends Plant Sci 22:961-975. https://doi.org/10. 1016/j.tplants.2017.08.011

da Cruz RP, Milach SCK (2004) Cold tolerance at the germination stage of rice: methods of evaluation and characterization of genotypes. Sci Agric 61:1-8. https://doi.org/10. 1590/S0103-90162004000100001

da Cruz RP, Sperotto RA, Cargnelutti D et al (2013) Avoiding damage and achieving cold tolerance in rice plants. Food Energy Secur 2:96-119. https://doi.org/10.1002/fes3.25

Dellaporta SL, Wood J, Hicks JB (1983) A plant DNA minipreparation: Version II. Plant Mol Biol Report 1:19-21. https://doi.org/10.1007/BF02712670

Di Rienzo JA, Guzmán AW, Casanoves F (2002) A multiplecomparisons method based on the distribution of the root node distance of a binary tree. J Agric Biol Environ Stat 7:129-142. https://doi.org/10.1198/10857110260141193

Fujino K, Matsuda Y (2010) Genome-wide analysis of genes targeted by qLTG3-1 controlling low-temperature germinability in rice. Plant Mol Biol 72:137-152. https://doi. org/10.1007/s11103-009-9559-x

Hospital F (2009) Challenges for effective marker-assisted selection in plants. Genetica 136:303-310. https://doi.org/ 10.1007/s10709-008-9307-1

Huang M, Guo Z (2005) Responses of antioxidative system to chilling stress in two rice cultivars differing in sensitivity. Biol Plant 49:81-84

INTA (2019) Estadísticas Temperaturas Mínimas. Instituto Nacional de Tecnología Agropecuaria Sistemas de Información Clima y Agua. https://climayagua.inta.gob.ar/ estadísticas_temperaturas_mínimas.

IRRI (2013) Standard evaluation system (SES) for rice, 5th edn. International Rice Research Institute, Los Baños, Philippines

Janmohammadi M, Zolla L, Rinalducci S (2015) Low temperature tolerance in plants: changes at the protein level. Phytochemistry 117:76-89

Kim SI, Tai TH (2011) Evaluation of seedling cold tolerance in rice cultivars: a comparison of visual ratings and quantitative indicators of physiological changes. Euphytica 178:437-447. https://doi.org/10.1007/s10681-010-0343-4

Kim S-I, Andaya VC, Tai TH (2011) Cold sensitivity in rice (Oryza sativa $\mathrm{L}$ ) is strongly correlated with a naturally occurring Ile99Val mutation in the multifunctional glutathione transferase isozyme GSTZ2. Biochem J. https:// doi.org/10.1042/BJ20101610

Krishnasamy V, Seshu DV (1989) Seed germination rate and associated characters in rice. Crop Sci 29:904. https://doi. org/10.2135/cropsci1989.0011183X002900040012x

Lancashire PD, Bleiholder H, Van Den BT et al (1991) A uniform decimal code for growth stages of crops and weeds. Ann Appl Biol 119:561-601. https://doi.org/10.1111/j. 1744-7348.1991.tb04895.x

Maguire JD (1962) Speed of germination-aid in selection and evaluation for seedling emergence and vigor1. Crop Sci 2:176. https://doi.org/10.2135/cropsci1962. 0011183X000200020033x

Mahender A, Anandan A, Pradhan SK (2015) Early seedling vigour, an imperative trait for direct-seeded rice: an overview on physio-morphological parameters and molecular markers. Planta 241:1027-1050. https://doi.org/10.1007/ s00425-015-2273-9

Martínez CP, Torres EA, Chatel M et al (2014) Rice breeding in Latin America. Plant Breed Rev 38:187-278. https://doi. org/10.1002/9781118916865.ch05

Mizoi J, Yamaguchi-Shinozaki K (2013) Molecular approaches to improve rice abiotic stress tolerance. In: Yang Y (ed) Rice protocols. Humana Press, Totowa, NJ, pp 269-283

Pachecoy MI, Ramirez IA, Marín A, Pontaroli AC (2014) Assessment of cold tolerance at early developmental stages and allelic variation at candidate genes in South American rice germplasm. Euphytica 197:423-434. https://doi.org/ 10.1007/s10681-014-1078-4

Ranal MA, de Santana DG (2006) How and why to measure the germination process? Rev Bras Botânica 29:1-11. https:// doi.org/10.1590/S0100-84042006000100002

Satoh T, Tezuka K, Kawamoto T et al (2016) Identification of QTLs controlling low-temperature germination of the East European rice (Oryza sativa L.) variety Maratteli. Euphytica 207:245-254. https://doi.org/10.1007/s10681015-1531-z

Shakiba E, Edwards JD, Jodari F et al (2017) Genetic architecture of cold tolerance in rice (Oryza sativa) determined through high resolution genome-wide analysis. PLoS ONE 12:e172133. https://doi.org/10.1371/journal.pone. 0172133

Sperotto RA, de Araújo Junior AT, Adamski JM et al (2017) Deep RNAseq indicates protective mechanisms of coldtolerant indica rice plants during early vegetative stage. Plant Cell Rep 37:1-29. https://doi.org/10.1007/s00299017-2234-9

Sthapit BR, Witcombe JR (1998) Inheritance of tolerance to chilling stress in rice during germination and plumule greening. Crop Sci 38:660-665

Tello-Ruiz MK, Naithani S, Stein JC et al (2018) Gramene 2018: unifying comparative genomics and pathway resources for plant research. Nucleic Acids Res 46:D1181D1189. https://doi.org/10.1093/nar/gkx1111

Van Ooijen G, Mayr G, Kasiem MMA et al (2008) Structurefunction analysis of the NB-ARC domain of plant disease 
resistance proteins. J Exp Bot 59:1383-1397. https://doi. org/10.1093/jxb/ern045

Wang J, Li H, Zhang L, Meng L (2012) User's manual of QTL Icimapping version 3.2. 2012) Quant Genet group, Inst Crop Sci Chinese Acad Agric Sci (CAAS), Beijing 100081, China Genet Resour Program CIMMYT, Mex p208 208.

Wang H, Lee A-R, Park S-Y et al (2018) Genome-wide association study reveals candidate genes related to low temperature tolerance in rice (Oryza sativa) during germination. 3 Biotech 8:235. https://doi.org/10.1007/ s13205-018-1252-9

Ye CA, Fukai SA, Godwin IA et al (2009) Cold tolerance in rice varieties at different growth stages. Crop Pasture Sci 60:328-338

Yoshida R, Kanno A, Kameya T (1996) Cool-temperature-lnduced chlorosis in rice plants. Plant Physiol 110:997-1005

Zhang Z-H, Yu S-B, Yu T et al (2005) Mapping quantitative trait loci (QTLs) for seedling-vigor using recombinant inbred lines of rice (Oryza sativa L.). F Crop Res 91:161-170. https://doi.org/10.1016/j.fcr.2004.06.004

Zhang Q, Chen Q, Wang S et al (2014) Rice and cold stress: methods for its evaluation and summary of cold tolerancerelated quantitative trait loci. Rice (N Y) 7:24. https://doi. org/10.1186/s12284-014-0024-3

Zhi-Hong Z, Li S, Wei L et al (2005) A major QTL conferring cold tolerance at the early seedling stage using recombinant inbred lines of rice (Oryza sativa L.). Plant Sci 168:527-534. https://doi.org/10.1016/j.plantsci.2004.09. 021

Publisher's Note Springer Nature remains neutral with regard to jurisdictional claims in published maps and institutional affiliations. 